\title{
以海藻酸钠为基体的 $\mathrm{Ag} / \mathrm{AgBr} / \mathrm{TiO}_{2}$ 整体式光催化剂的 高效制备及其光催化性能
}

\author{
童 琴 ${ }^{1}$ ，董亚梅 ${ }^{1}$ ，严 良 ${ }^{1}$ ，何丹农 ${ }^{1,2}$
}

(1. 纳米技术及应用国家工程研究中心, 上海 200241 ; 2. 上海交通大学 材料科学与工程学院, 上海 200240)

摘 要: 以海藻酸钠为基体制备出尺寸均一的整体式光催化剂 $\mathrm{SA}-\mathrm{Ag} / \mathrm{AgBr} / \mathrm{TiO}_{2}$, 通过 SEM、TEM、HRTEM、XPS、 FT-IR 以及 BET 等手段对产物形貌、结构、比表面积和光学性质进行表征，并测定了其对罗丹明 B(RhB)溶液的光 催化性能。结果表明, 整体式光催化剂 $\mathrm{SA} 12-\mathrm{Ag} / \mathrm{AgBr} / \mathrm{TiO}_{2}\left(\mathrm{Ag} / \mathrm{AgBr} / \mathrm{TiO}_{2}\right.$ 催化剂的质量分数为 $\left.12 \%\right)$ 的结构稳定, 且 具有良好的光催化活性，即在紫外光照 $60 \mathrm{~min}$ 内, $\mathrm{RhB}$ 溶液降解完全，在可见光照 $120 \mathrm{~min}$ 下 $\mathrm{RhB}$ 的降解率可达到 $54.1 \%$, 且在 5 次重复试验中仍保持 $96 \%$ 以上的催化活性，稳定性良好。这种整体式光催化剂有效解决了粉体材料 难回收、二次污染和固定化后光催化效率低等问题。

关 键 词: 海藻酸钠; $\mathrm{Ag} / \mathrm{AgBr} / \mathrm{TiO}_{2}$ 复合光催化剂; 整体式光催化剂; 可见光

中图分类号: 0643 文献标识码: A

\section{High-efficient Synthesis and Photocatalytic Properties of $\mathrm{Ag} / \mathrm{AgBr} / \mathrm{TiO}_{2} \mathrm{Mono}$ - lithic Photocatalysts Using Sodium Alginate as Substrate}

\author{
TONG Qin ${ }^{1}$, DONG Ya-Mei ${ }^{1}$, YAN Liang ${ }^{1}$, HE Dan-Nong ${ }^{1,2}$
}

(1. National Engineering Research Center for Nanotechnology, Shanghai 200241, China; 2. School of Materials Science and Engineering, Shanghai Jiao Tong University, Shanghai 200240, China)

\begin{abstract}
SA}-\mathrm{Ag} / \mathrm{AgBr} / \mathrm{TiO}_{2}$ monolithic photocatalysts with uniform particle size distribution was synthesized by using sodium alginate as a substrate. Its structure and morphology were characterized by XRD, XPS, SEM, TEM, and HRTEM. The optical properties and the surface area of the $\mathrm{SA}-\mathrm{Ag} / \mathrm{AgBr} / \mathrm{TiO}_{2}$ monolithic photocatalysts were characterized by FT-IR and BET. The as-prepared photocatalyst with good stability exhibited enhanced photocatalytic performance for degradation of RhB solution under both UV and visible light. The $\mathrm{SA}-\mathrm{Ag} / \mathrm{AgBr} / \mathrm{TiO}_{2}$ monolithic photocatalyst with $12 \mathrm{wt} \% \mathrm{Ag} / \mathrm{AgBr} / \mathrm{TiO}_{2}$ display optimum properties i.e. about $100 \%$ degradation rate for the RhB solution for 60 min under UV irradiation and $54.1 \%$ degradation rate for the $\mathrm{RhB}$ solution under visible light irradiation for $120 \mathrm{~min}$. Besides, after 5 cycles, the RhB degradation rate still keeps higher than 96\%, showing an excellent reusability of the photocatalysts. The as-prepared SA-Ag/ $\mathrm{AgBr} / \mathrm{TiO}_{2}$ monolithic photocatalysts may effectively solve the problems including photocatalytic activity, secondary pollution and photocatalyst recycling.
\end{abstract}

Key words: sodium alginate; $\mathrm{Ag} / \mathrm{AgBr} / \mathrm{TiO}_{2}$ photocatlyst; monolithic photocatalysts; visible light

收稿日期: 2016-08-29; 收到修改稿日期：2016-11-03

基金项目：国家重大科学研究计划(2015CB931902)；上海市青年科技启明星计划(B 类)(16QB1402200) National Basic Research Program of China (2015CB931902); Shanghai Science and Technology Development Funds (16QB1402200)

作者简介: 童 琴(1986-), 女, 硕士研究生. E-mail: tongqin_7557@163.com

通讯作者: 何丹农, 教授. E-mail: hdn_nercn@163.com 
$\mathrm{AgBr}$ 的禁带宽度为 $2.6 \mathrm{eV}$, 是一种具有很强光 敏性的无机半导体材料, 能够在可见光下表现出很 高的光响应能力, 利用这个特性, 将其与 $\mathrm{TiO}_{2}$ 复合, 可以将 $\mathrm{TiO}_{2}$ 的响应光谱拓展到可见光范围, 从而提 高 $\mathrm{TiO}_{2}$ 对太阳光的利用效率, 这也为实际应用提供 了可能性 ${ }^{[1-3]}$ 。现有的光催化剂材料大多以悬浮态存 在, 虽然具有较高的降解效率, 但是反应后催化剂 回收困难, 不仅影响出水水质, 而且随着反应的连 续循环, 需要不断地补充催化剂, 导致处理成本的 增加。为了解决这个问题, 很多研究者力图将催化 剂粉末固定化在其他基底材料上, 以便解决催化剂 回收难和易流失等问题，降低成本，这对实际生产 中大规模应用和推广具有重大意义。目前国内外有 关光催化剂粉末的固定化载体及方法有很多, 常见 的用于固定化的材料有钛板 ${ }^{[4]}$ 、高分子聚合物 ${ }^{[5]}$ 、 玻璃纤维网 ${ }^{[6]}$ 、分子篮 ${ }^{[7]} 、$ 陶瓷 $^{[8]}$ 、纤维素 ${ }^{[9]}$ 以及粘 土 $^{[10]}$ 等, 采用溶胶-凝胶法 ${ }^{[11]}$ 、溅射法 ${ }^{[12]}$ 、电沉积 法 $^{[13]}$ 和粘结剂法 ${ }^{[14]}$ 等, 不仅可以解决催化剂分离回 收问题, 还可以克服催化剂粉末稳定性差和易中毒 的缺点, 并且适用于活性组分和载体的各种功能的 组合来设计催化反应器, 有利于大规模的水处理。

例如, $\mathrm{Kim}$ 等 ${ }^{[15]}$ 曾将 $\mathrm{TiO}_{2}$ 光催化剂用硅胶固定; Radeka 等 ${ }^{[16]}$ 将介孔 $\mathrm{TiO}_{2}$ 材料涂覆在粘土瓦片的表 面上, 并在紫外光下降解对氯苯甲酸。近年来, 智能 型水凝胶因其独特的物理结构和性质在纳米粒子载 体领域中逐步得到研究者的重视。杨娜等 ${ }^{[17]}$ 采用掺 杂法制备具有光催化性能的聚( $\mathrm{N}$-异丙基丙烯酰胺)/ 纳米 $\mathrm{TiO}_{2}$ 温敏复合凝胶, 利用凝胶的温敏性有效提 高了对活性蓝 X-BR 的降解率, 并改善了纳米 $\mathrm{TiO}_{2}$ 的可回收性。Haraguchi 等 ${ }^{[18]}$ 以 N-异丙基丙烯酰胺 为单体, 以粘土作为物理交联剂, 通过原位自由基 聚合制备出新型聚 $\mathrm{N}$-异丙基丙烯酰胺/粘土纳米利 水凝胶, 表现出良好的强度和韧性。如果将光催化 性能较好的纳米 $\mathrm{TiO}_{2}$ 基光催化粉末固定于具有优 异物理和化学性质的水凝胶中, 可以有效解决纳米 催化剂粉末的回收问题，同时凝胶的孔结构降低了 其凝胶对纳米光催化剂的催化性能的阻碍作用, 实 现两种材料的性能互补。

本工作针对粉末状光催化剂回收困难和负载型 催化剂催化活性的问题, 采用水凝胶法制备整体型 光催化剂, 即以海藻酸钠为基体制备的 SA 系列整 体式光催化剂, 通过对其进行 XRD、SEM、XPS、

FT-IR、BET 等表征及对 RhB 水溶液的光催化降解 实验, 系统研究了 $\mathrm{SA}$ 系列整体式光催化剂的光催 化性能。

\section{1 实验方法}

\section{$1.1 \mathrm{Ag} / \mathrm{AgBr} / \mathrm{TiO}_{2}$ 光催化剂的制备}

称取 6.0 g F123(聚环氧乙烷-聚环氧丙烷-聚环 氧乙烷三嵌段共聚物)加入到 $60 \mathrm{~mL}$ 无水乙醇中, 溶 解后加入 $7.0 \mathrm{~mL}$ 钛酸四丁酯, 搅拌 $1 \mathrm{~h}$ 。然后缓慢 滴加 $30 \mathrm{~mL}$ 去离子水, 使钛酸四丁酯充分水解。反 应完毕后，将该混合物转移至水热釜中，反应 $24 \mathrm{~h}$, 控温 $150^{\circ} \mathrm{C}$ 。用去离子水和无水乙醇清洗沉淀数次, 干燥研磨后在 $350^{\circ} \mathrm{C}$ 煅烧 $4 \mathrm{~h}$, 即可得到白色的介孔 $\mathrm{TiO}_{2}$ 纳米光催化剂。

将盛有 $0.4 \mathrm{~g}$ 介孔纳米 $\mathrm{TiO}_{2}$ 光催化剂、 $\mathrm{CTAB}$ 和 $200 \mathrm{~mL}$ 去离子水的反应器置于超声器中, 超声 $15 \mathrm{~min}$ 后再搅拌 $30 \mathrm{~min}$, 使其混合均匀。再称取与 $\mathrm{CTAB}$ 相应质量的 $\mathrm{AgNO}_{3}$ 加入到该混合溶液中, 摚 拌 $30 \mathrm{~min}$, 混合均匀后转移至水热釜中, 反应 $12 \mathrm{~h}$, 控温 $150^{\circ} \mathrm{C}$ 。用去离子水和无水乙醇清洗若干次后, $60^{\circ} \mathrm{C}$ 干燥 $6 \mathrm{~h}$ 后研磨备用。最后, 将该粉末放置在氙 灯 $(220 \mathrm{~V}, 500 \mathrm{~W})$ 下照射 $120 \mathrm{~min}$, 使生成的 $\mathrm{AgBr}$ 中 部分 $\mathrm{Ag}^{+}$在氙灯的光照下还原成 $\mathrm{Ag}^{0}$, 即可得到 $\mathrm{M}-\mathrm{TiO}_{2}$ 负载 $\mathrm{AgBr}$ 的 $\mathrm{Ag} / \mathrm{AgBr} / \mathrm{TiO}_{2}$ 复合光催化剂。

\section{2 整体式 $\mathrm{SA}-\mathrm{Ag} / \mathrm{AgBr} / \mathrm{TiO}_{2}$ 光催化剂的制备}

室温下, 称取 $1.5 \mathrm{~g}$ 海藻酸钠粉末加入到 $46.5 \mathrm{~mL}$ 去离子水中, $60^{\circ} \mathrm{C}$ 下加热摚拌 $2 \mathrm{~h}$ 。待完全溶解后, 向其中加入 $2 \mathrm{~g} \mathrm{Ag} / \mathrm{AgBr} / \mathrm{TiO}_{2}$ 复合光催化剂粉末, 继续摚拌 $2 \mathrm{~h}$ 使其均匀分散在海藻酸钠中。配置浓 度为 $10 \%$ 的 $\mathrm{CaCl}_{2}$ 溶液 $100 \mathrm{~mL}$, 将上述分散有光催 化剂的海藻酸钠体系滴加至其中, 形成均匀的小 球。滴加完毕后, 将小球从 $\mathrm{CaCl}_{2}$ 溶液中分离出来, 即可得到质量分数为 $4 \%$ 的以海藻酸钠为基体的整 体式光催化剂(SA4- $\left.\mathrm{Ag} / \mathrm{AgBr} / \mathrm{TiO}_{2}-\mathrm{NCs}\right)$ 。

重复上述步骤，调整去离子水和 $\mathrm{Ag} / \mathrm{AgBr} / \mathrm{TiO}_{2}$ 复合光催化剂的添加量，分别制备质量分数为 $8 \%$ 和 $12 \%$ 的以海藻酸钠为基体的整体式光催化剂(SA8$\mathrm{Ag} / \mathrm{AgBr} / \mathrm{TiO}_{2}-\mathrm{NCs}$ 和 $\left.\mathrm{SA} 12-\mathrm{Ag} / \mathrm{AgBr} / \mathrm{TiO}_{2}-\mathrm{NCs}\right)$ 。

\section{3 结构表征}

用日本理学 $\mathrm{D} / \mathrm{max}-2600 / \mathrm{PC} \mathrm{X}$ 射线多晶体转靶 衍射仪(XRD)对样品进行晶相分析; 用日立 S-4800 型场发射扫描电子显微镜(SEM)观察产物形貌; 用 Thermo Fisher Scientific Nicolet 6700 型傅里叶变换 红外光谱仪对产物进行红外光谱分析; 用铂金埃尔 默仪器 Lambda 950 型紫外可见近红外分光光度计 对产物紫外-可见光吸收性能进行研究; 用美国麦 克公司的 ASAP 2020M 型比表面及孔径分析仪 
(BET)对产物的比表面积进行表征。

\section{4 整体式 $\mathrm{SA}-\mathrm{Ag} / \mathrm{AgBr} / \mathrm{TiO}_{2}$ 光催化剂的光 催化性能}

称取整体式 $\mathrm{SA} 12-\mathrm{Ag} / \mathrm{AgBr} / \mathrm{TiO}_{2}$ 光催化剂 $0.1 \mathrm{~g}$, 分散至 $100 \mathrm{~mL}$ 浓度为 $1 \times 10^{-5} \mathrm{~mol} / \mathrm{L}$ 的 $\mathrm{RhB}$ 溶液中, 分别在 $500 \mathrm{~W}$ 高压录灯和 $500 \mathrm{~W}$ 氙灯下进行光催化 降解实验。为了确保光催化剂在光照前已达到吸附脱附平衡, 降解前先进行 $30 \mathrm{~min}$ 的暗处理, 取不同 时间点的溶液测定其对紫外光的吸收强度, 并与不 同质量分数的 $\mathrm{SA} 4-\mathrm{Ag} / \mathrm{AgBr} / \mathrm{TiO}_{2}$ 和 $\mathrm{SA} 8 \mathrm{-Ag} / \mathrm{AgBr} /$ $\mathrm{TiO}_{2}$ 的降解结果进行对比。

\section{2 结果与讨论}

\section{1 产物的形貌与结构}

图 1 是 $\mathrm{Ag} / \mathrm{AgBr} / \mathrm{TiO}_{2}$ 纳米晶的 TEM 和 HRTEM 照片。从图 1(a)可以看到, $\mathrm{Ag} / \mathrm{AgBr} / \mathrm{TiO}_{2}$ 纳米晶为近 球形的纳米粒子, 粒径尺寸均一, 而且在一些介孔 $\mathrm{TiO}_{2}$ 小球的表面负载有 $\mathrm{AgBr}$ 颗粒, 这从 HRTEM 数 据中也可以进一步得到验证。在图 1(b)中可以清楚 地观察到三种差异较为明显的晶格条纹, 通过测量 分析可以发现, 三种晶格条纹的晶面间距分别为 $0.235 、 0.243$ 和 $0.333 \mathrm{~nm}$, 分别对应着 $\mathrm{Ag}$ 的(100)

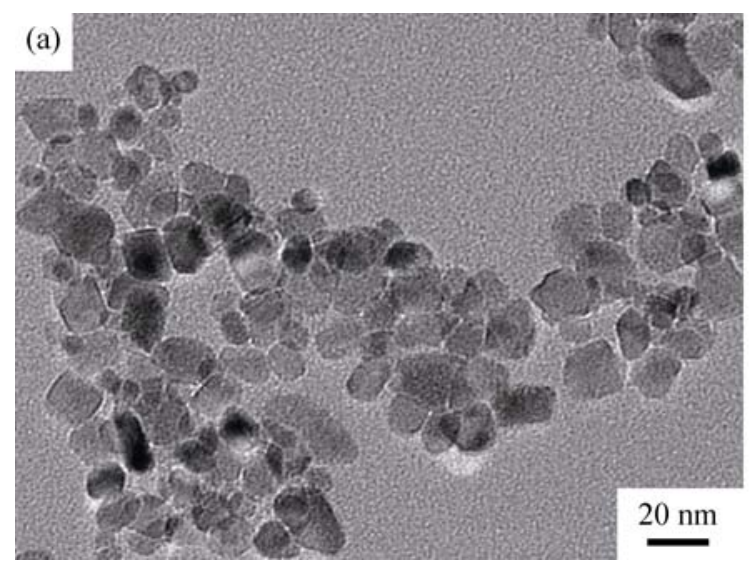

图 $1 \mathrm{Ag} / \mathrm{AgBr} / \mathrm{TiO}_{2}$ 纳米晶的 TEM(a) 和 $\mathrm{HRTEM}(\mathrm{b})$ 照片

Fig. 1 TEM (a) and HRTEM (b) images of $\mathrm{Ag} / \mathrm{AgBr} / \mathrm{TiO}{ }_{2} \mathrm{NCs}$
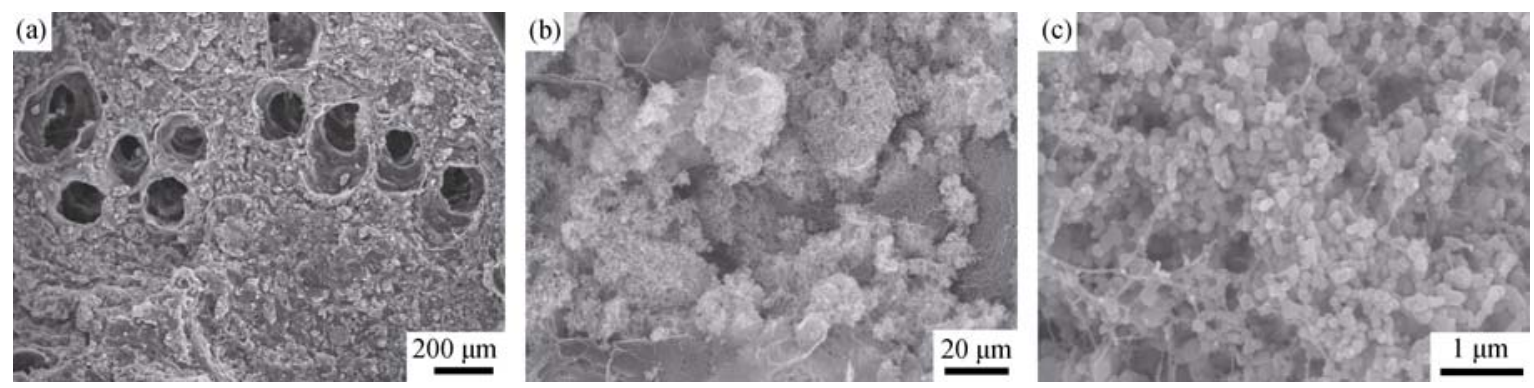

图 2 以海藻酸钠为基体的整体式 $\mathrm{Ag} / \mathrm{AgBr} / \mathrm{TiO}_{2}$ 光催化剂的不同倍率 $\mathrm{SEM}$ 照片

Fig. 2 Different magnification SEM images of the $\mathrm{SA}-\mathrm{Ag} / \mathrm{AgBr} / \mathrm{TiO}_{2}$ monolithic photocatalysts 


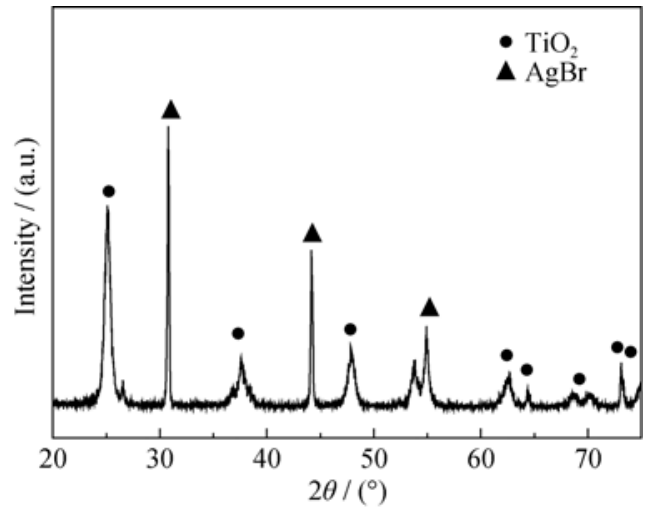

图 $3 \mathrm{Ag} / \mathrm{AgBr} / \mathrm{TiO}_{2}$ 纳米晶的 XRD 图谱

Fig. 3 XRD pattern of $\mathrm{Ag} / \mathrm{AgBr} / \mathrm{TiO}_{2} \mathrm{NCs}$

\subsection{XPS 分析}

为了进一步探究 $\mathrm{Ag} / \mathrm{AgBr} / \mathrm{TiO}_{2}$ 复合光催化剂 以及元素 $\mathrm{Ag}$ 的存在形态, 采用 $\mathrm{X}$ 光电子能谱对该 纳米晶的化学组成和表面特性进行分析。图 4 为 $\mathrm{Ag} / \mathrm{AgBr} / \mathrm{TiO}_{2}$ 纳米晶以及 $\mathrm{Ag}$ 的 $3 \mathrm{~d}$ 轨道的 XPS 能 谱图。从图 4(a)可以看到, 该样品表面的化学组成 是 $\mathrm{Ti} 、 \mathrm{O} 、 \mathrm{Ag} 、 \mathrm{Br}$ 元素, 其中 $\mathrm{Ag}$ 的 $3 \mathrm{~d}$ 轨道的高分 辨率扫描 XPS 光谱图如图 4(b)所示, 从图中可以看到 两个主峰的位置大概在结合能为 373.5 和 $367.5 \mathrm{eV}$ 处, 分别对应于 $\mathrm{Ag}$ 的 $3 \mathrm{~d} 3 / 2$ 和 $3 \mathrm{~d} 2 / 5$, 而且在两个主峰 的旁边各自还有一个较小的峰, 即在 374.1 和 $368.2 \mathrm{eV}$ 的位置上。其中, 两个主峰 373.5 和 $367.5 \mathrm{eV}$ 来自 于 $\mathrm{AgBr}$ 中的 $\mathrm{Ag}^{+}$, 而 374.1 和 $368.2 \mathrm{eV}$ 来自于 $\mathrm{Ag}^{0[20]}$ 。XPS 结果进一步说明 $\mathrm{Ag} / \mathrm{AgBr} / \mathrm{TiO}_{2}$ 复合光 催化剂中确实有 $\mathrm{Ag}^{0}$, 这与 XRD 和 HRTEM 数据结 果相一致。

\section{3 光学性质}

图 5 为以海藻酸钠为基体的整体式 $\mathrm{Ag} / \mathrm{AgBr}$ / $\mathrm{TiO}_{2}$ 复合光催化剂的 FT-IR 图谱, 从图中可以看到, 在 3440、2920、1620、1416、1350 和 $1150 \mathrm{~cm}^{-1}$ 等

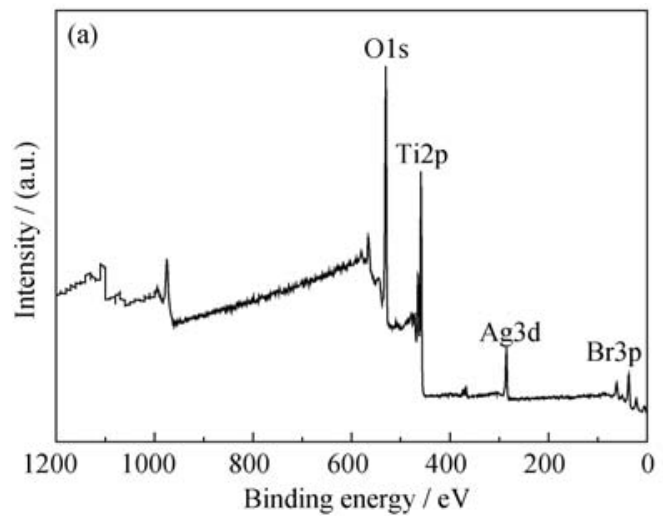

处都出现了不同强度的吸收峰。其中, $3440 \mathrm{~cm}^{-1}$ 处 的吸收峰对应为 $\mathrm{O}-\mathrm{H}$ 的伸缩振动 ${ }^{[21]} ; 2920 \mathrm{~cm}^{-1}$ 左右 出现的小峰则是由于于海藻酸钠的糖单元中六元 环的存在产生张力而导致的 $\mathrm{C}-\mathrm{H}$ 伸缩振动 ${ }^{[22]}$; $1620 \mathrm{~cm}^{-1}$ 和 $1416 \mathrm{~cm}^{-1}$ 处的特征吸收峰分别对应羧 基(-COO-) 的不对称伸缩振动和对称伸缩振动 ${ }^{[23]}$; 而 1350 和 $1150 \mathrm{~cm}^{-1}$ 处则分别对应与 $\mathrm{C}-\mathrm{O}-\mathrm{H}$ 弯曲振 动和环醚糖苷键中 $\mathrm{C}-\mathrm{O}-\mathrm{C}$ 的伸缩振动。随着 $\mathrm{Ag} / \mathrm{AgCl} / \mathrm{TiO}_{2}$ 复合光催化剂质量分数的增加, 在 $3440 \mathrm{~cm}^{-1}$ 处的伸缩振动峰明显变宽了, 这是由于 $\mathrm{TiO}_{2}$ 纳米光催化剂表面含有大量羟基, 能够与海藻 酸钠中的羟基相互作用形成氢键, 从而使 $\mathrm{O}-\mathrm{H}$ 的伸 展振动减弱。而催化剂的添加量越大, 形成的氢 键越多, $\mathrm{O}-\mathrm{H}$ 的伸展振动减弱就越明显, 从而使 $3440 \mathrm{~cm}^{-1}$ 处的吸收峰越宽。

\subsection{BET 分析}

不同 $\mathrm{Ag} / \mathrm{AgBr} / \mathrm{TiO}_{2}$ 催化剂含量的海藻酸钠型整 体式复合光催化剂 $\mathrm{SA}-\mathrm{Ag} / \mathrm{AgBr} / \mathrm{TiO}_{2}$ 的比表面积测量 结果显示: 当 $\mathrm{Ag} / \mathrm{AgBr} / \mathrm{TiO}_{2}$ 光催化剂的含量为 $4 \mathrm{wt} \%$ 时, 以海藻酸钠为基体的整体式复合光催化剂的比 表面积为 $8.6096 \mathrm{~m}^{2} / \mathrm{g}$; 当 $\mathrm{Ag} / \mathrm{AgBr} / \mathrm{TiO}_{2}$ 光催化剂的 含量为 $8 \mathrm{wt} \%$ 时, 整体式复合光催化剂比表面积为 $42.5217 \mathrm{~m}^{2} / \mathrm{g}$; 而当 $\mathrm{Ag} / \mathrm{AgBr} / \mathrm{TiO}_{2}$ 光催化剂的含量增加 到 $12 \mathrm{wt} \%$ 时, 比表面积也随之增加到 $53.5845 \mathrm{~m}^{2} / \mathrm{g}$ 。这 是由于光催化剂的比表面积比水凝胶基体大得多, 随着光催化剂的比例增加, 整体式光催化剂的比表 面积也相应增加，这会在很大程度上促进催化反应 速率，从而提高其催化性能。

\section{5 光催化活性分析}

不同 $\mathrm{SA}-\mathrm{Ag} / \mathrm{AgBr} / \mathrm{TiO}_{2}-\mathrm{NCs}$ 催化剂含量的整体 式光催化剂, 分别在紫外光和可见光下对 $\mathrm{RhB}$ 溶液 进行降解，其光催化降解曲线如图 6 所示。从图 6(a)

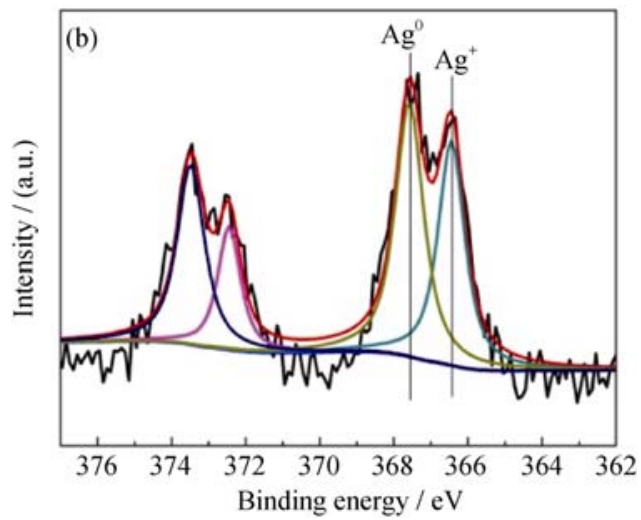

图 $4 \mathrm{Ag} / \mathrm{AgBr} / \mathrm{TiO}_{2}$ 纳米晶(a)和 $\mathrm{Ag} 3 \mathrm{~d}(\mathrm{~b})$ 的 XPS 图谱

Fig. 4 XPS spectra of $\mathrm{Ag} / \mathrm{AgBr} / \mathrm{TiO}_{2} \mathrm{NCs}$ (a) and $\mathrm{Ag} 3 \mathrm{~d}$ (b) 


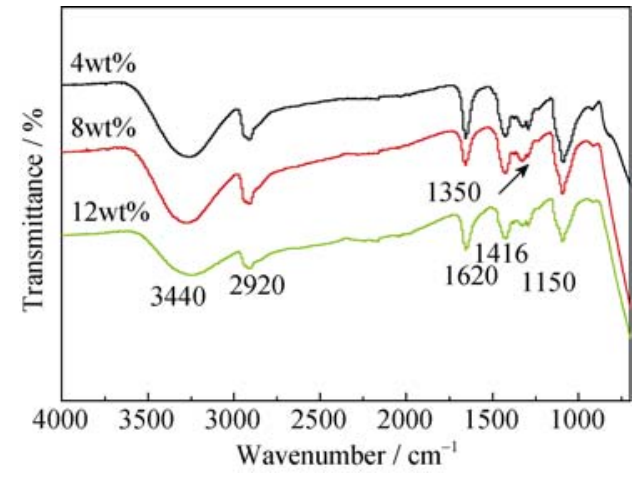

图 5 不同 $\mathrm{Ag} / \mathrm{AgBr} / \mathrm{TiO}_{2}$ 催化剂含量的海藻酸钠型整体式光 催化剂的 FT-IR 图谱

Fig. 5 FT-IR spectra of the $\mathrm{SA}-\mathrm{Ag} / \mathrm{AgBr} / \mathrm{TiO}{ }_{2}$ monolithic photocatalysts with different contents of $\mathrm{Ag} / \mathrm{AgBr} / \mathrm{TiO}_{2}$

中可以看到, 随着复合光催化剂的添加量的增加, $\mathrm{SA}-\mathrm{Ag} / \mathrm{AgBr} / \mathrm{TiO}_{2}-\mathrm{NCs}$ 整体式光催化剂的活性也相 应增强, 在紫外光照 $60 \mathrm{~min}$ 内, $\mathrm{RhB}$ 溶液几乎可以完 全降解。在可见光照射下, $\mathrm{SA} 12-\mathrm{Ag} / \mathrm{AgBr} / \mathrm{TiO}_{2}-\mathrm{NCs}$ 整体式光催化剂的催化效率比 $4 \mathrm{wt} \%$ 和 $8 \mathrm{wt} \%$ 的样品 要好, 光照约 $120 \mathrm{~min}$, 降解率达到 $54.1 \%$, 具有良 好的可见光光催化活性。

为进一步测试整体式光催化剂 $\mathrm{SA} 12-\mathrm{Ag} /$ $\mathrm{AgBr} / \mathrm{TiO}_{2}$ 的稳定性, 对实验完毕的 $\mathrm{SA} 12-\mathrm{Ag} / \mathrm{AgBr} /$ $\mathrm{TiO}_{2}$ 进行回收, 并在可见光照射下对 $\mathrm{RhB}$ 溶液进行光 催化降解实验。经循环使用发现, $\mathrm{SA} 12-\mathrm{Ag} / \mathrm{AgBr} / \mathrm{TiO}_{2}$ 光催化剂的催化效率同比下降了 $1.5 \%$, 重复使用 2 次, 整体式光催化剂的催化效率相比原来下降了 $3.0 \%$ 。经 5 次循环使用后, 该光催化剂对 RhB 溶液的光催化降 解能力并没有出现显著的大幅度下降, 均保持在原来 活性的 96\%以上, 展现出良好的光催化活性。这也说 明利用本研究的固定化方法制备得到的整体式光催
化剂具有较高的结构稳定性, 能够循环再利用。

\section{3 结论}

采用海藻酸钠为基体制备出的整体式光催化剂 $\mathrm{SA} 12-\mathrm{Ag} / \mathrm{AgBr} / \mathrm{TiO}_{2}$ 不仅结构稳定, 而且在紫外光 和可见光下均展现出良好的光催化活性, 即在紫外 光照 $60 \mathrm{~min}$ 内, $\mathrm{RhB}$ 溶液就可以降解完全, 在可见 光照 $120 \mathrm{~min}$ 下 $\mathrm{RhB}$ 的降解率可达到 $54.1 \%$ 。通过 本研究的固定化法合成的整体式光催化剂在 5 次重 复试验中仍保持 $96 \%$ 以上的催化活性, 稳定性良好, 有效解决了粉体材料难以回收、二次污染和固定化 后光催化效率低等问题, 表现出相当好的稳定性, 而且制备工艺简单，可以实现工程化应用。

\section{参考文献:}

[1] LIU QIANG, ZHANG ZHI-BO, DONG CHANG-QING, et al. Improved visible-light photocatalytic activity of Bi-crystalline mesoporous titania codoped with carbon and silver. Journal of Inorganic Materials, 2014, 29(12): 1333-1338.

[2] WANG HUI-LEI, LIU XIAO-HENG. Preparation of silver nanoparticle loaded mesoporous $\mathrm{TiO}_{2}$ and its photocatalytic property. Journal of Inorganic Materials, 2016, 31(5): 555-560.

[3] WANG XIAO-PING, TANG YU-XIN, CHEN ZHONG, et al. Highly stable heterostructured $\mathrm{Ag}-\mathrm{AgBr} / \mathrm{TiO}_{2}$ composite: a bifunctional visible-light active photocatalyst for destruction of ibuprofen and bacteria. Journal of Materials Chemistry, 2012, 22(43): 23149-23158.

[4] MAI LI-XIANG, WANG DA-WEI, ZHANG SHENG, et al. Synthesis and bactericidal ability of $\mathrm{Ag} / \mathrm{TiO}_{2}$, composite films deposited on titanium plate. Applied Surface Science, 2010, 257(3): 974-978.

[5] LANGLET M, KIM A, AUDIER M, et al. Sol-Gel preparation of photocatalytic $\mathrm{TiO}_{2}$, films on polymer substrates. Journal of Sol-Gel Science and Technology, 2002, 25(3): 223-234.
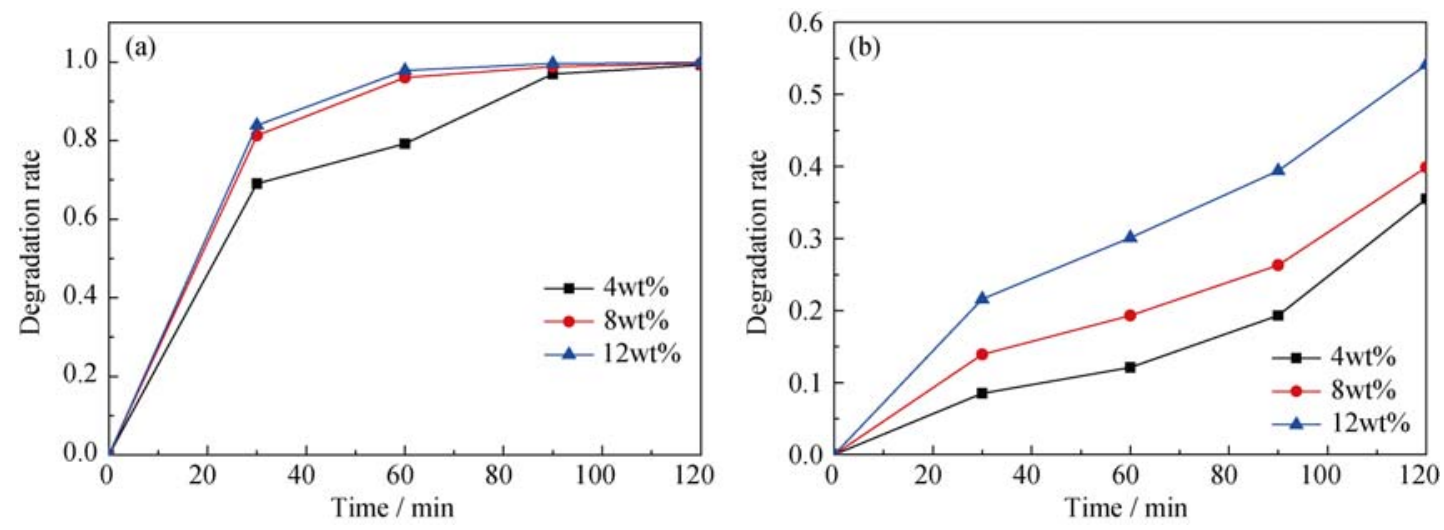

图 6 不同 $\mathrm{Ag} / \mathrm{AgBr} / \mathrm{TiO}_{2}$ 催化剂含量的海藻酸钠型整体式催化剂 $\mathrm{SA}-\mathrm{Ag} / \mathrm{AgBr} / \mathrm{TiO}$ 在紫外光(a)和 可见光(b)下对 $R h B$ 光催化降解曲线

Fig. $6 \mathrm{RhB}$ photocatalytic degradation by the $\mathrm{SA}-\mathrm{Ag} / \mathrm{AgBr} / \mathrm{TiO}_{2}$ monolithic photocatalysts with different contents of $\mathrm{Ag} / \mathrm{AgBr} / \mathrm{TiO}_{2}$ under UV irradiation (a) and visiblelight irradiation (b) 
[6] CHEN LIN, YANG SU-DONG, MÄDER EDITH, et al. Controlled synthesis of hierarchical $\mathrm{TiO}_{2}$ nanoparticles on glass fibres and their photocatalytic performance. Dalton Transactions, 2014, 43(33): 12743-12753.

[7] FENG LI-LI, ZHAO WEI, LIU YANG, et al. Photocatalytic degradation of Rhodamine $\mathrm{B}$ by nanocrystalline $\mathrm{TiO}_{2}$ loaded into MCM-41 molecular sieves. Acta Phys. -Chim. Sin., 2009, 25(07): 1347-1351.

[8] SU HUA-ZHI, PENG CHENG, WU JIAN-QING. A review of titanium dioxide photocatalytic material and its application in ceramics. Foshan Ceramics, 2016, 1(234): 1-9.

[9] ORTELli S, BLOSI M, ALBONETTI S, et al. $\mathrm{TiO}_{2}$ based nano-photocatalysis immobilized on cellulose substrates. Journal of Photochemistry \& Photobiology A Chemistry, 2013, 276: 58-64.

[10] RANOGAJEC JONJAUA, RADEKA MIROSLAVA, BAČKALIĆ ZORAN, et al. Photocatalytic and superhydrophilic phenomena of $\mathrm{TiO}_{2}$ coated clay roofing tiles. Chemical Industry \& Chemical Engineering Quarterly, 2010, 16(2): 117-126.

[11] WU SU-JUAN, WANG CONG, CUI YIN-FANG, et al. BiOCl nano/microstructures on substrates: Synthesis and photocatalytic properties. Materials Letters, 2011, 65(9): 1344-1347.

[12] YANG MYUNG, HONG SEONG-HYEON. Gas sensing property of ITO/ $\mathrm{SnO}_{2}$ hetero-structure deposited by RF magnetron sputter. Journal of Electrochemical Society, 2010, 157(11): J392-J396.

[13] CORDER-ARIAS LUIS, BOCCACCINI ALDO R, VIRTANEN SANNAKAISA. Electrochemical behavior of nanostructured $\mathrm{TiO}_{2} /$ alginate composite coating on magnesium alloy AZ91D via electrophoretic deposition. Surface \& Coatings Technology, 2015, 265: 212-217.

[14] LI YOU-JI, LI XIAO-DONG, LI JUN-WEN, et al. Effects of active carbon carrier on phase transform and crystallite growth of titanium dioxide in $\mathrm{TiO}_{2} / \mathrm{AC}$. Journal of Inorganic Materials, 2005, 20(2): 291-298.
[15] KIM DONG SEOG, PARK YOUNG SEEK. Photocatalytic decol-orization of Rhodamine B by immobilized $\mathrm{TiO}_{2}$ onto silicone sealant. Chemical Engineering Journal, 2006, 116(2): 133-137.

[16] RADEKA M, MARKOV S, LONČAR, E, et al. Photocatalytic effects of $\mathrm{TiO}_{2}$, mesoporous coating immobilized on clay roofing tiles. Journal of the European Ceramic Society, 2014, 34(1): 127-136.

[17] YANG NA, LIU YI-JUN, FENG YUN-FANG. Preparation and characterization of thermosensitive photocatalyst: poly (N-isopropylacrylamide)/Nano- $\mathrm{TiO}_{2}$ hydrogel. Journal of Zhejiang Institute of Science and Technology, 2010, 27(4): 543-547.

[18] HARAGUCHI KAZUTOSHI, TAKEHISA TORU, FAN SIMON. Effects of clay content on the properties of nanocomposite hydrogels composed of poly(N-isopropylacrylamide) and clay. Macromolecules, 2002, 35(27): 10162-10171.

[19] HOU YANG, LI XIN-YONG, ZHAO QI-DONG, et al. $\mathrm{TiO}_{2}$ nanotube/ $\mathrm{Ag}-\mathrm{AgBr}$ three-component nanojunction for efficient photoconversion. Journal of Materials Chemistry, 2011, 21(44): 18067-18076.

[20] YANG YU-XIN, GUO WAN, GUO YING-NA, et al. Fabrication of Z-scheme plasmonic photocatalyst $\mathrm{Ag} @ \mathrm{AgBr} / \mathrm{g}-\mathrm{C}_{3} \mathrm{~N}_{4}$, with enhanced visible-light photocatalytic activity. Journal of Hazardous Materials, 2014, 271c(5): 150-159.

[21] YU YI-JUN, ZHI-HUAI SI, CHEN SHUANG-JUN, et al. Facile synthesis of polyaniline-sodium alginate nanofibers. Langmuir, 2006, 22(8): 3899-3905.

[22] LE-TIEN CANH, MILLETTE MATHIEU, LACROIX MONIQUE, et al. Modified alginate matrices for the immobilization of bioactive agents. Biotechnology \& Applied Biochemistry, 2004, 39(2): 189-198.

[23] HUANG R Y M, PAL R, MOON G Y. Characteristics of sodium alginate membranes for the pervaporation dehydration of ethanol-water and isopropanol-water mixtures. Journal of Membrane Science, 1999, 160(1): 101-113. 